\title{
Treatment of Gaucher disease with an enzyme inhibitor
}

\author{
NORMAN S. RADIN \\ Mental Health Research Institute and Division of Nephrology MSRBII, University of Michigan, 1550 W. \\ Medical Centre Drive Ann Arbor, MI 48109-0676, USA
}

Received 23 May 1995, revised 20 June 1995

\begin{abstract}
The hypothesis is offered predicting that Gaucher patients could be treated with a drug that slows the synthesis of glucosylceramide, the lipid that accumulates in this disorder. The present therapeutic approach involves augmenting the defective enzyme, glucosylceramide $\beta$-glucosidase, with exogenous $\beta$-glucosidase isolated from human tissue. This spectacularly expensive mode of treatment should be replaceable with a suitable enzyme inhibitor that simply slows formation of the lipid and matches the rate of synthesis with the rate of the defective, slowly working $\beta$ glucosidase. Several drugs that possess this ability are available, the best known of which is 1-phenyl-2-

decanoylamino-3-morpholino-1-propanol (PDMP), a designer inhibitor that resembles the synthase's substrate and product. PDMP has been found to be effective in mice, rats, fish, and a wide variety of cultured cells. Its use, at suitable dosages, seems to be harmless, although long-term tests have not been made. The lack of suitable animal models of Gaucher disease has made it difficult to test the hypothesis adequately, but PDMP does rapidly lower the levels of glucosylceramide in normal animal tissues and the animals evidently do well with the lowered levels of glucosylceramide and its more complex glycolipid metabolites.
\end{abstract}

Keywords: glucosylceramidase insufficiency, Gaucher disease chemotherapy, glucosylceramide synthase inhibition, PDMP (1-phenyl-2-decanoylamino-3-morpholino-1-propanol)

Abbreviations: PDMP, 1-phenyl-2-decanoylamino-3-morpholino-1-propanol; GlcCer, glucosylceramide; i.p., intraperitoneal.

\section{Introduction}

Most Gaucher patients possess a modest level of glucosylceramide $\beta$-glucosidase, as much as $40 \%$ of the normal level, so their disorder develops slowly. The slowness of development is evidently due to a small imbalance between the rate of glucosylceramide (GlcCer) synthesis and the rate of its hydrolysis. This results in the gradual accumulation of GlcCer. The clinical symptoms seem to arise mainly from accumulated macrophages, which apparently form in response to the problem of disposing of the GlcCer derived from catabolizing red and white blood cells. Injection of chemically modified $\beta$ glucosidase ('Ceredase') is the present treatment modality, since it seeks out the cells that store or normally catabolize the lipid. However it must be injected throughout the life of the patient and constitutes an extraordinarily expensive, albeit effective, way to treat these patients. The hypothesis developed in this paper is that slowing the synthesis of GlcCer with a suitable inhibitor will allow the patient to form red and white blood cells that have a lowered content of GlcCer. The GlcCer in these cells should then be able to undergo hydrolysis at an adequate rate, despite the patient's low level of glucosidase. In addition, the glycolipid presently stored before treatment is begun, will either be utilized for normal cell functioning or hydrolysed. The storage cells themselves probably also synthesize GlcCer for their own needs; this must add to the burden but could be slowed with a synthase inhibitor.

White cells synthesize GlcCer, probably throughout their life span. Thus the administration of a synthase inhibitor would rapidly lower the content of GlcCer in circulating cells, producing a rapid reduction in the rate of glycolipid deposition.

By now, $\sim 50$ papers have been published describing the effectiveness of D-threo-1-phenyl-2-decanoylamino-3morpholino-1-propanol (PDMP) and related compounds as inhibitors of GlcCer synthase. The compounds are active at the micromolar level in mice, rats, fish, and a wide variety of cultured cells (canine and human kidney, human tumours, $\mathrm{C} 3 \mathrm{H}$ murine splenocytes, IL2-dependent 
CTLL, rabbit skin fibroblasts, murine Lewis lung carcinoma, A431 human epidermoid carcinoma, human and mouse embryonal carcinoma, human intestinal epithelial, NIH 3T3, B16 melanoma, multiple drug resistant and others). PDMP is easy to synthesize so it should be a relatively economical substitute or adjuvant for Ceredase. It is currently available from four companies for non-human use. This paper presents some of the evidence supporting the proposed rationale. Related, newer drugs, more effective in cultured cell studies, are also described.

\section{Mode of action}

PDMP was designed to simulate the structures of ceramide (the lipoidal precursor of GlcCer) and GlcCer itself. It apparently acts at the enzyme's active site, as well as on a similar binding site [1]. It is not a covalently inactivating inhibitor, but some evidence [2] points to partial metabolic conversion to such a substance. Its use in mice leads to some elevation in GlcCer glucosidase [3], an effect which should enhance its usefulness, assuming such an effect can be obtained with the defective glucosidase.

The optical enantiomer of PDMP, the L-threo form, does not inhibit GlcCer synthase, but its use in some cultured cells has led to a reduction in glycolipid levels. Thus, for reasons of economy, the DL-racemic mixture may be satisfactory in Gaucher patients. It has been used in some of the published studies of PDMP.

\section{Metabolism of PDMP}

Tests with labelled PDMP in mice have shown that it is largely destroyed and then excreted within $-3 \mathrm{~h}$ after a single dose i.p. [2]. The destruction is evidently due to a cytochrome $\mathrm{P} 450$, since it can be slowed or blocked by coadministration of either of two P450 inhibitors possessing low toxicity, cimetidine and piperonyl butoxide. Most of the animal studies were done without the aid of a P450 inhibitor, so a high dose was necessary to produce a lasting effect. The labelled drug exhibited good penetration of all organs tested and should thus be effective throughout the patient's body. Preliminary tests indicated that PDMP readily enters the body when mixed with food, thus obviating the need for injections.

\section{Effectiveness in lowering GlcCer levels}

A high but tolerable dose of DL-PDMP (100 $\left.\mathrm{m} \mathrm{kg}^{-1}\right)$, injected i.p. into normal mice, produced a $35 \%$ loss of GlcCer in kidney (as well as a 15\% loss of the next higher metabolite, lactosylceramide) within just $5 \mathrm{~h}$ [3]. This rapid decrease shows that GlcCer undergoes rapid metabolism in normal kidney, possibly in all organs. In mice injected twice a day for $4 \mathrm{~d}$, a similar decrease in kidney GlcCer was found.

Coadministration of L-cycloserine augmented the effect of PDMP in the latter experiment. (L-Cycloserine is not the antitubercular drug, D-cycloserine.) The combination lowered the level of the glycolipid by $50 \%$. L-Cycloserine is an inhibitor of sphingosine synthesis, leading to a depletion of its product, ceramide [4]. Ceramide competes against PDMP in binding to the synthase [1]. Thus cycloserine potentiates the effectiveness of PDMP in two ways and might prove a useful adjuvant in Gaucher patients.

When PDMP was added to the growth medium of embryonic medaka, a tiny freshwater fish, the embryos hatched normally by $10 \mathrm{~d}$ [5]. The treated fish were morphologically normal. This long exposure to $20 \mu \mathrm{M}$ PDMP resulted in a drastic loss of the ability to synthesize GlcCer and its higher metabolites from $\left[{ }^{3} \mathrm{H}\right]$ galactose and a virtually complete loss of the reactivity of several glycolipids with specific antibodies. Since nearly all glycolipids are derived from GlcCer, blocking the synthesis of GlcCer leads to catabolic depletion of all these compounds.

After hatching, the fish were raised in normal medium for 2 weeks, during which time there was restoration of the ability to make glycolipids. The PDMP-treated fish exhibited normal swimming, feeding, responses to light and obstacle avoidance.

\section{Is PDMP toxic?}

An experiment with normal young mice treated with high doses of PDMP, given in 12 daily injections, and analysed $40 \mathrm{~h}$ after the last injection, showed that the body, liver, brain, and spleen weights were normal, although the kidneys were $11 \%$ smaller (statistically significant) [6]. Initially there was a loss in body weight, but the treated mice caught up with the saline controls. Blood cell counts of the RBC, monocytes, lymphocytes, eosinophiles, and neutrophiles revealed no abnormalities.

The size of kidneys in mice seems to be particularly sensitive to the level of GlcCer. Injecting testosterone into young mice rapidly raised the level of GlcCer synthase, lowered the level of GlcCer $\beta$-glucosidase, and accelerated the rate of kidney growth [7]. This finding suggests that the enlargement of liver and spleen characteristic of Gaucher disease reflects, in part, a proliferative action of GlcCer. Intraperitoneal injection of GlcCer into mice did indeed result in deposition in the liver and rapid growth of the liver [8]. (Predictably, these effects disappeared in a few days because the glucosidase was not defective in these animals.) 
The shrinking action of PDMP was utilized in diabetic rats, which - like human diabetics - develop enlarged kidneys. The PDMP treatment brought the kidney size back to normal despite the high level of glucose [9]. Normal kidney function was retained. Curiously, Gaucher patients do not seem to show kidney abnormalities or deposition of GlcCer in their kidneys. Perhaps their kidneys excrete accumulated GlcCer.

In other tests, in which mice were first inoculatec with Ehrlich ascites carcinoma cells, PDMP given for 5 or 10 days greatly prolonged the survival time for many of the mice and apparently produced permanent cures in $\sim 30 \%$ of the animals [6]. Control inoculated mice all died within $\sim 24$ days. When the surviving mice, still frisky after 135 days, were reinoculated with new ascites cells, the tumours failed to develop. Evidently the mice had developed protective antibodies against the tumour cells. This is suggestive (but incomplete) evidence that the drug does not harm the immune system; if anything, it augments the ability of the immune system to attack tumour cells. These observations are of special importance to Gaucher patients, who have a tendency to develop some forms of cancer [10], possibly due to a proliferative action of GlcCer.

The medaka fish mentioned above seemed to exhibit no toxic reaction to PDMP despite their great depletion of GlcCer. In addition, B16 melanoma cells cultured in $\sim 20 \mu \mathrm{M}$ PDMP also lost most of their GlcCer, yet recovered their synthetic abilities when placed in a drugfree medium [11].

The lack of toxicity in cells and organisms depleted of their normal level of GlcCer is surprising, considering the almost ubiquitous distribution of glycolipids. In the case of the laboratory cells, mice, rats, and fish, the environment was of course optimal and unstressful. From the many reports of glycolipid functions in cell binding, growth, and differentiation, one would guess that mammals living in the real world would be in a precarious state if they were too depleted of their glycolipids. On the other hand, chronic depletion might protect us from attack by many viruses and bacteria, which are known to bind to specific GlcCer-derived glycolipids. Such protective action by PDMP against binding of cultured human kidney and intestinal cells to $E$. coli has been demonstrated [12].

\section{Side-effects of PDMP}

PDMP and related inhibitors can, at sufficiently high concentrations, drastically slow growth and proliferation. This is apparently the result of a process causing accumulation of GlcCer's precursor, ceramide [13]. This substance has recently come to be considered a part of cell signalling events leading to cell death. However the inhibitors do not produce ceramide accumulation at low
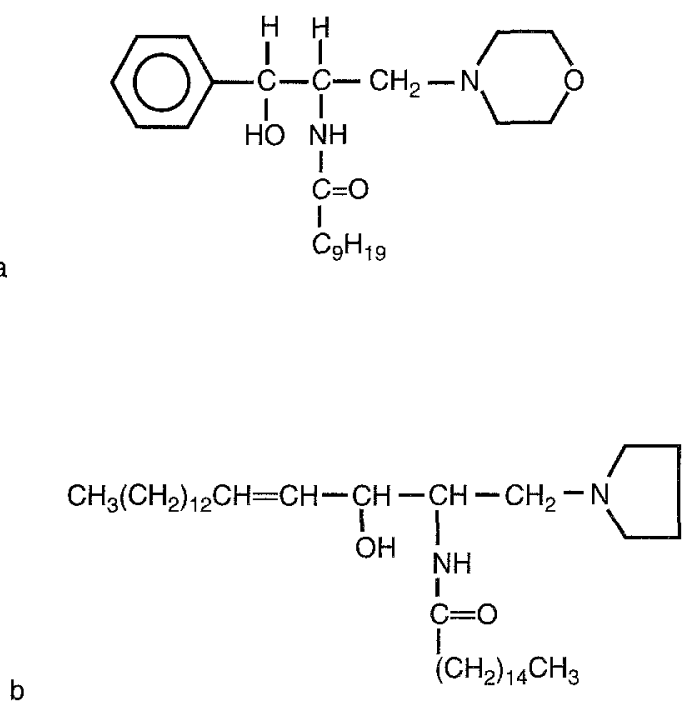

Figure 1. (a) Structure of D-threo-PDMP; (b) pyrrolidino deoxyceramide.

concentrations, yet readily block GlcCer synthesis. The very effective aliphatic inhibitor, pyrrolidino deoxyceramide (Fig. 1b), does not cause ceramide accumulation or growth inhibition even at higher concentrations, so it might be safer than PDMP.

Soon after injection of high doses into mice and rats, behavioural symptoms were seen, subsiding after about 15 min. Including PDMP in mouse feed produced no observable reactions. Of course bolus type doses would be unnecessary in human subjects.

Mice injected with DL-PDMP nine times were found to 1 lose $23 \%$ of their kidney ceramide level and $31 \%$ of their kidney GlcCer, while sphingomyelin was unaffected [3]. In another experiment, using two injections, mice developed elevated levels in the specific activity of GlcCer glucosidase $(+25 \%)$, GalCer galactosidase $(+34 \%)$, and sphingomyelinase $(+33 \%)$. $\beta$-Glucuronidase was slightly reduced.

Cultured cells have been analysed in depth and changes were found in a variety of cell components [13-15]. It is interesting that ceramide levels went up (at high dosage levels), in contradiction to the decrease seen in intact mouse kidney. It seems likely that some of the changes seen in cultured cells do not occur in intact animals, owing to compensatory systems. However, like any other drug, PDMP could exhibit surprises for treated patients.

\section{Other potentially useful mediators of GlcCer metabolism}

A single injection of the tranquilizer, chlorpromazine, into mice was found to lower GlcCer synthase specific activity in liver substantially $(-47 \%)$ in only $3 \mathrm{~h}$, while producing 
an increase in ceramide concentration $(+25 \%)$ [16]. The mechanism for the effect is not known but the possible application to Gaucher disease warrants further study.

A search for activators of Gaunher glucocerebrosidase might prove useful. The enzyme in the brain of a Gaucher patient was tripled in activity, in vitro, by gluconoyl hydrazine [17]. A series of syntheses of related structures ought to be attempted.

PDMP homologues made with longer chain fatty acids (PDMP contains the decanoic acid moiety) have been found to inhibit GlcCer synthase in vitro more effectively than PDMP. Preliminary tests of the longer chain compounds in mice inoculated with Ehrlich ascites cells indicated it was more effective in curing the mice [6].

Recent work has shown that replacing the morpholine moiety with other cyclic amines enhances the effectiveness against GlcCer synthase and tumour cell growth [13]. Some aliphatic analogues of PDMP have also proved highly effective as synthase inhibitors. As little as $0.1 \mu \mathrm{M}$ inhibitor with cultured cells produced distinct depletion of GlcCer in $24 \mathrm{~h}$. It seems likely that we can produce an effective depletion of glycolipids in Gaucher patients with just small doses of these compounds. Here too further synthetic efforts could furnish even more effective drugs.

Recently, a cationic glycolipid with only a short aliphatic chain ( $N$-butyl-deoxynojirimicin) was found to be a good inhibitor of GlcCer synthase. The authors proposed that this, like PDMP, might be useful for Gaucher patients. An isomeric form of this substance has been reported to be more effective, while avoiding inhibition of glucosidases [18]. Replacing the rather nonspecific butyl group with a ceramide-like group might greatly enhance its inhibitory activity.

A study of GlcCer glucosidase [19] has revealed that the enzyme has the ability to utilize various alcohols instead of water (i.e. it can carry out transglucosylation). It seems possible that ingesting a suitable glucose acceptor would allow even a Gaucher patient's enzyme to convert GlcCer to a more soluble, more easily excreted glucoside. This approach looks useful and warrants further study.

Some normal body components are involved in the action of glucosidase: saposin C (a small protein that acts as a cofactor in the action of the enzyme) and phosphatidylserine, which has a similar, coordinate function. Supplementation by these substances or synthetic compounds related in structure might prove very beneficial. We have shown that mice preloaded with GlcCer and treated with the glucosidase inhibitor, conduritol B epoxide ('temporary Gaucher mice'), can be restored to normalcy more rapidly if injected with phosphatidylserine [20].

Cultured cells absorb glucosidase much better if the enzyme is first complexed with saposin $\mathrm{C}$ and phospha- tidylserine [21]. This characteristic might be helpful with Ceredase for lowering the required dosage.

\section{Possible complications}

(a) The proposed hypothesis assumes that our GlcCer synthase inhibitor acts on circulating blood cells and the cells that form the blood elements. These are the cells that furnish much of the burden of GlcCer hydrolysis on the liver and spleen. Many types of cells are sensitive to these compounds - mammalian and piscine, in organ masses and separate, in culture dishes. Thus it seems very plausible to expect that the blood cells are also sensitive. The same assumption applies to the macrophages that store, and presumably also synthesize, GlcCer.

(b) Perhaps a cell type in the body exists that is exceptionally sensitive to the synthase inhibitors. Kidney cells do seem to be more sensitive but not toxically so. Multiple drug resistant cells are somewhat more sensitive to synthase inhibitors [22] but this is hardly a negative property. Obviously there is a need for long-term toxicity testing in animals.

(c) Perhaps a patient would develop tolerance toward the synthase inhibitors. This is always a potential problem with drugs that must be used over a long period. Rosenwald and Pagano were able to develop a strain of CHO cells that required double the dose of PDMP to achieve the same effect [22]. Only tests in human subjects will tell us if this is a realistic problem.

(d) The toxicity of the proposed adjuvant drugs (e.g. Lcycloserine) also needs more study. An advantage of using a combination of drugs is that the dose of each could be lowered, compared with single drug use. The combinations could include Ceredase at a more economical level.

(e) The glycolipid-depleted blood cells in inhibitortreated Gaucher patients might not function well; perhaps their life spans would be shortened. However mice injected with high doses of PDMP for 12 days seemed perfectly healthy.

(f) The hypothesis presented here would not be useful for the infantile, neuronopathic form of Gaucher disease since there is so little functioning glucosidase in these patients.

\section{References}

1. Inokuchi J, Radin NS (1987) J Lipid Res 28: 565-71.

2. Shukla A, Radin NS (1991) J Lipid Res 32: 713-22.

3. Shukla G, Shukla A, Inokuchi J, Radin NS (1991) Biochim Biophys Acta 1083: 101-8.

4. Sundaram KS, Lev M (1984) $J$ Neurochem 42: 577-81.

5. Fenderson BA, Ostrander GK, Hausken Z, Radin NS, Hakomori S (1992) Exp Cell Res 198: 36266.

6. Inokuchi J, Mason I, Radin NS (1987) Cancer Lett 38: 2330. 
7. Shukla A, Shukla GS, Radin NS (1992) Am J Physiol 262: F24-29.

8. Datta SC, Radin NS (1988) Lipids 23: 508-10.

9. Zador IZ, Deshmukh GD, Kunkel R, Johnson K, Radin NS, Shayman, JA (1993) J Clin Invest 91: 797-803.

10. Shiran A, Bremner B, Laor A, Tatarsky I (1993) Cancer 72: 219-24.

11. Inokuchi J, Momosaki $\mathrm{K}$, Shimeno $\mathrm{H}$, Nagamatsu A, Radin NS (1989) J Cell Physiol 141: 573-83.

12. Svensson M, Lindstedt R, Radin NS, Svanborg C (1994) Infect Immun 62: 4404-10.

13. Abe A, Radin NS, Shayman JA, Wotring LL, Zipkin RE, Sivakumar R, Ruggieri JM, Carson KG, Ganem B (1995) $J$ Lipid Res 36: 611-21.

14. Radin NS, Shayman JA, Inokuchi J (1993) In Advances in Lipid Research: Sphingolipids in Signaling, Part B (Bell RM, Hannun YA, Merrill AH, eds), Vol. 28, pp. 183-213. Orlando:
Academic Press.

15. Radin NS, Shayman JA (1993) In NeuroProtocols: A Companion to Methods in Neurosciences (Fisher SK, Bleasdale JE, eds), Vol. 3, pp. 145-55. Orlando: Academic Press.

16. Hospattankar V, Vunnam RR, Radin NS (1982) Lipids 17: $538-43$.

17. Daniels LB, Coyle PJ, Glew RH, Radin NS, Labow RS (1982) Arch Neurol 39: 550-56.

18. Platt FM, Neises GR, Karlsson GB, Dwek RA, Butters TD (1994) $J$ Biol Chem 269: 27108-14.

19. Vanderjagt DJ, Fry DE, Glew RH (1994) Biochem J 300: 309-15.

20. Datta SC, Radin NS (1988) Biochem Biophys Res Commun 152: $155-60$.

21. Datta SC, Snider RM, Radin NS (1986) Biochim Biophys Acta 877: $387-98$.

22. Rosenwald AG, Pagano RE (1994) J Lipid Res 35: $1232-40$. 